\title{
Probing Thermal-induced Phonon Energy Shift of SiC in Nanoscale by in situ Vibrational Spectroscopy
}

\author{
Xingxu Yan ${ }^{1}$, Chengyan Liu ${ }^{1}$, Chaitanya A. Gadre ${ }^{2}$, Ruqian $\mathrm{Wu}^{2}$ and Xiaoqing Pan ${ }^{1,2,3}$ \\ 1. Department of Materials Science and Engineering, University of California - Irvine, Irvine, CA, USA. \\ 2. Department of Physics and Astronomy, University of California - Irvine, Irvine, CA, USA. \\ 3. Irvine Materials Research Institute (IMRI), University of California - Irvine, Irvine, CA, USA.
}

High spatial resolution temperature measurement is crucial to investigating the thermal transport and, heat dissipation in nanodevices and nanotechnologies. Plasmon resonance has successfully applied to detect temperature gradients in Al by electron energy-loss spectroscopy (EELS) in a scanning transmission electron microscope (STEM) [1]. Due to the thermal-induced lattice expansion and associated change of valence electron density, the energy value of plasmon peak redshifts as temperature increases. However, for the materials with low coefficient of thermal expansion, a plasmon-based thermometer cannot provide precise temperature measurement. In addition to plasmons, phonons also exhibit a temperature-dependent energy shift. Taking advantage of the newly designed monochromator and spectrometer, vibrational spectroscopy can be realized in an advanced STEM with sub-10 meV energy resolution [2-4]. Combined with the high spatial resolution of STEM imaging, both bulk and surface phonon modes can be distinguished with different spatial distribution [4]. Furthermore, temperature-dependence of phonon modes can be employed to measure the local temperature by principle of detailed balance [5, 6], and should provide more fundamental physical information such as thermal expansion of crystals, and anharmonic effects at high temperature. Here, we investigate thermal behavior of phonon mode in SiC nanoparticle by in-situ vibrational spectroscopy.

$\mathrm{SiC}$ is a wide-bandgap semiconductor with high rigidity, superior chemical inertness, large thermal conductivity, a low thermal expansion coefficient, and is widely used in power electronics. In our experiment, $\mathrm{SiC}$ nanoparticles were dispersed on the Protochips Fusion ${ }^{\mathrm{TM}}$ thermal chip, which is compatible with Nion's cartridge design. Vibrational spectra were obtained by Nion UltraSTEM 200 equipped with $\mathrm{C} 3 / \mathrm{C} 5$ aberration corrector and high-energy resolution monochromated EELS system (HERMES). In order to optimize the signal intensity and maintain reasonable energy resolution, each EELS measurement consists of 50 200 frames with 1s exposure time for each frame, which are then aligned by the zero loss peak (ZLP) center. Then, background subtraction is performed by fitting an exponential function [4].

Fig. 1a depicts a $20 \mathrm{~nm} \mathrm{SiC} \mathrm{nanoparticle} \mathrm{(NP)} \mathrm{with} \mathrm{3C} \mathrm{phase} \mathrm{lying} \mathrm{on} \mathrm{a} \mathrm{thin} \mathrm{carbon} \mathrm{film.} \mathrm{EEL} \mathrm{spectra}$ were acquired under interior (inside of specimen) and aloof (outside of specimen) configuration as shown in Fig. 1. The interior spectrum is separated into three sub-peaks at 99.2, 113.9 and $120.0 \mathrm{meV}$, which are attributed to the standard transversal optical (TO, $100 \mathrm{meV})$, surface phonon polariton (SPhP, $116 \mathrm{meV})$ and longitudinal optical mode ( $\mathrm{LO}, 120 \mathrm{meV})$, respectively [7]. Interestingly, the aloof spectrum doesn't have any signal in this energy range. The dipole scattering between surface mode and fast electron beam is suppressed in the case of nanoparticle due to the dramatically reduced surface area. Fig. 2a shows the temperature-dependence of bulk phonon, by plotting the peak centers of the TO modes between room temperature and $1000^{\circ} \mathrm{C}$. It was found that the energy shift is proportional to the change of temperature with the slope of $-3.5 \mathrm{meV} / 1000 \mathrm{~K}$. Molecular dynamic (MD) simulations of the TO mode show a similar energy shift of $-4.9 \mathrm{meV} / 1000 \mathrm{~K}$ (Fig. 2b). Detailed analysis figures out that the effect of lattice expansion 
contributes to an energy shift only by $-0.75 \mathrm{meV} / 1000 \mathrm{~K}$, whereas the contribution of the anharmonic effect is expectedly as high as $-4.32 \mathrm{meV} / 1000 \mathrm{~K}$. This result provides a dedicated interpretation of the thermal behavior of phonon, and paves the way for probing local temperature by optical phonon based thermometers [8].

References:

[1] M Mecklenburg et al., Science 347 (2015), p. 629.

[2] OL Krivanek et al., Nature 514 (2014), p. 209.

[3] P Rez et al., Nat. Commun. 7 (2016), p. 10945.

[4] MJ Lagos et al., Nature 543 (2017), p. 529.

[5] JC Idrobo et al., Phys. Rev. Lett. 120 (2018), p. 095901.

[6] MJ Lagos and PE Batson, Nano Lett. 18 (2018), p. 4556.

[7] M Dayan, Surf. Sci. 149 (1985), p. L33.

[8] This work was supported by the Department of Energy (DOE) under Grant DE-SC0014430. TEM experiments was conducted using the facilities in the Irvine Materials Research Institute (IMRI) at the University of California-Irvine.
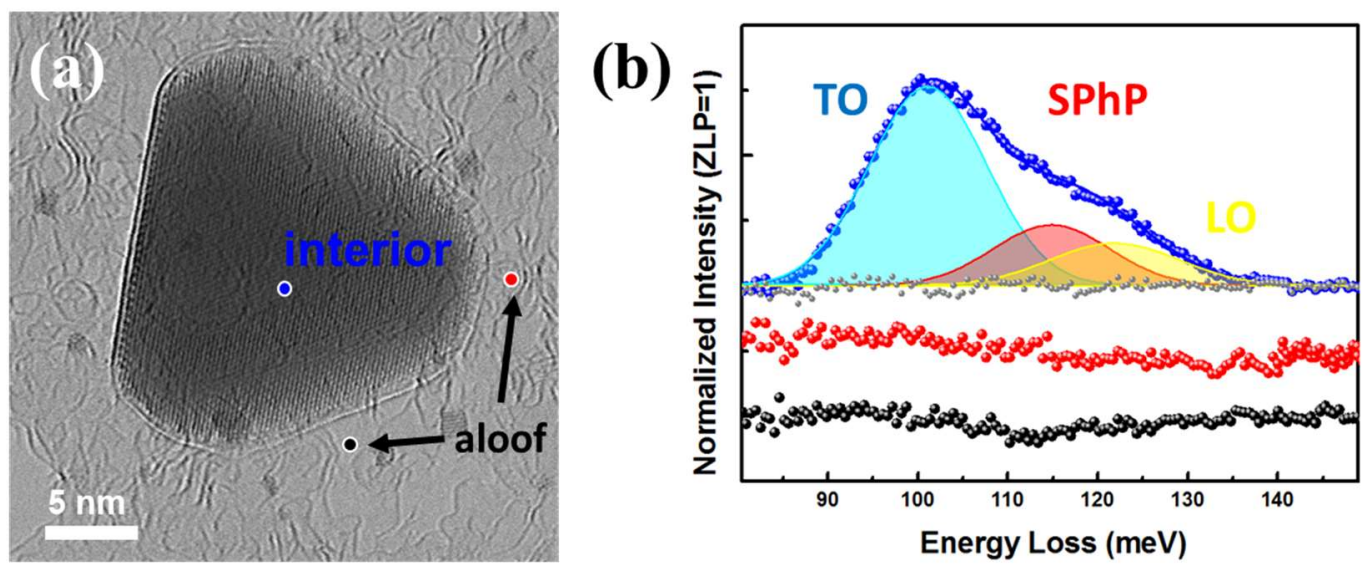

Figure 1. Vibrational spectra on a SiC NP with the 3C phase. (a) STEM-BF image of a NP with one marker for interior position and two additional markers for aloof positions which are just outside the nanoparticle. (b) Three background-subtracted vibrational spectra from three positions in (a).
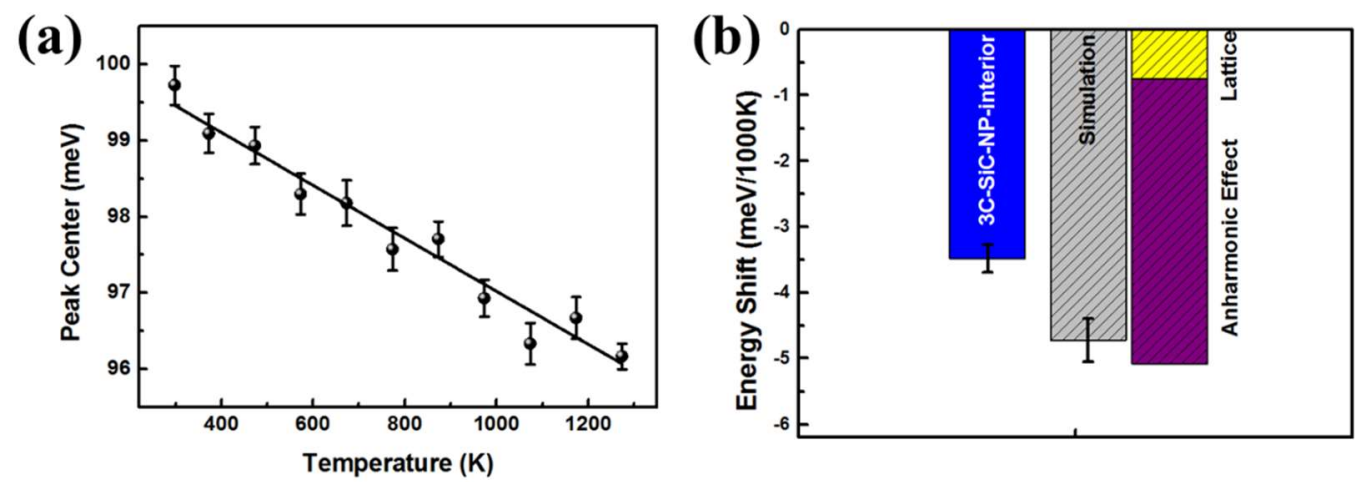

Figure 2. Temperature-dependence of optical phonon mode. (a) The peak center of energy loss as a function of temperature, along with fitting line. (b) Comparison of energy shift of phonon mode from experiment and simulation. The 'Lattice' and 'Anharmonic Effect' labels represent the estimated energy shifts of optical TO mode solely due to lattice expansion effect and anharmonic effect, respectively. 